\begin{tabular}{|c|c|c|c|c|}
\hline \multicolumn{5}{|c|}{ НАУЧНО-ТЕХНИЧЕСКИЙ ВЕСТНИК ИНФОРМАЦИОННЫХ ТЕХНОЛОГИЙ, МЕХАНИКИ И ОПТИКИ } \\
\hline & $\begin{array}{ll}\text { январь-февраль } 2015 \quad \text { Том } 15 \text { № } 1\end{array}$ & ISSN 2226-1494 & http://ntv.ifmo.ru/ & 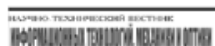 \\
\hline УНИВЕРСИТЕТ ИТМО & SCIENTIFIC AND TECHNICAL JOURNAL OF INF & ION TECHNOLOG & HANICS AND OPTICS & \\
\hline
\end{tabular}

\author{
УДК 621.382.038 \\ ИЗУЧЕНИЕ МЕХАНИЗМОВ, ОТВЕТСТВЕННЫХ ЗА ДЕГРАДАЦИЮ \\ ЭФФЕКТИВНОСТИ СВЕТОДИОДОВ НА ОСНОВЕ НИТРИДОВ ТРЕТЬЕЙ ГРУППЫ \\ Н.М. Шмидт ${ }^{a}$, А.С. Усиков ${ }^{\text {b, }}$, Е.И. Шабунина ${ }^{a}$, А.Е. Черняков ${ }^{a}$, С.Ю. Курин ${ }^{d}$ Ю.Н. Макаров ${ }^{\text {, d }}$, \\ Х.И. Хелава ${ }^{\mathrm{b}}$, Б.П. Папченко \\ ${ }^{\mathrm{a}}$ ФТИ им. А.Ф. Иоффе, Санкт-Петербург, 194021, Российская Федерация \\ ${ }^{\mathbf{b}}$ Нитридные кристаллы Inc-DP, Дир Парк, NY 11729, США \\ ' Университет ИТМО, Санкт-Петербург, 197101, Россия \\ d ООО «Группа компаний «Нитридные кристаллы», Санкт-Петербург, 194156, Российская Федерация \\ Адрес для переписки: Natalia. Shmidt@mail.ioffe.ru \\ Информация о статье \\ Поступила в редакцию 04.12 .14 , принята к печати 20.12 .14 \\ doi: $10.17586 / 2226-1494-2015-15-1-46-53$ \\ Язык статьи - русский \\ Ссылка для цитирования: Шмидт Н.М., Усиков А.С., Шабунина Е.И., Черняков А.Е., Курин С.Ю., Макаров Ю.Н., Хелава Н.И., \\ Папченко Б.П. Изучение механизмов, ответственных за деградацию эффективности светодиодов на основе нитридов третьей груп- \\ пы // Научно-технический вестник информационных технологий, механики и оптики. 2015. Том 15. № 1. С. 46-53
}

Аннотация. Приведены результаты исследования деградации внешней квантовой эффективности двух типов светодиодов, ультрафиолетового и синего диапазонов, на основе полупроводниковых нитридов третьей группы. Кратко рассмотрены известные механизмы, приводящие к деградации внешней квантовой эффективности светодиодов при старении. Применение комплекса методов для изучения светодиодов после разных временных стадий старения позволило впервые выяснить возможность участия механизма многофононной рекомбинации носителей в дефектообразовании под действием инжекционного тока в системе протяженных дефектов и в локальных областях с флуктуациями состава твердого раствора. Этот комплекс включает: анализ вольтамперных характеристик, в том числе при напряжениях менее 2 В; методики низкочастотного шума; инфракрасную микроскопию. Многофононная рекомбинация носителей сопровождается генерацией собственных дефектов, в том числе атомов индия и галлия в междоузлиях, и их миграцией. Эти процессы приводят к изменению свойств системы протяженных дефектов и локальных областей твердого раствора, уменьшая концентрацию неравновесных носителей, участвующих в излучательной рекомбинации, что приводит к деградации внешней квантовой эффективности. Показано, что этот механизм может участвовать в деградации внешней квантовой эффективности светодиодов как ультрафиолетового, так и синего диапазона. В рамках этого механизма находит объяснение немонотонный характер развития процесса деградации и катастрофические отказы этих светодиодов, а также низкие сроки службы ультрафиолетовых светодиодов.

Ключевые слова: светодиоды на основе нитридов третьей группы, система протяженных дефектов, механизмы дефектообразования, деградация.

Благодарности. Работа проводится Университетом ИТМО при финансовой поддержке государства в лице Минобрнауки России в рамках соглашения № 14.575.21.0054 (уникальный идентификатор прикладных научных исследований RFMEFI57514X0054) о предоставлении субсидии по теме: «Исследование новых технических возможностей для создания экологически чистого генератора водорода с использованием фотоэлектрохимического элемента на основе наноструктур полупроводниковых нитридов третьей группы».

\title{
STUDY OF MECHANISMS RESPONSIBLE FOR THE EFFICIENCY DEGRADATION OF THE III-NITRIDES LIGHT EMITTING DIODES
}

N.M. Shmidt ${ }^{a}$, A.S. Usikov ${ }^{b, c}$, E.I. Shabunina ${ }^{a}$, A.E. Chernyakov ${ }^{a}$, S.Yu. Kurin ${ }^{d}$, Yu. N. Makarov ${ }^{b, d}$, H. Helava ${ }^{b}$, B.P. Papchenko ${ }^{\mathrm{c}}$

${ }^{a}$ Ioffe Institute, Saint Petersburg, 194021, Russian Federation

${ }^{b}$ Nitride Crystals Inc-DP, Deer Park, NY 11729, USA

c ITMO University, Saint Petersburg, 197101, Russian Federation

${ }^{\text {d }}$ Nitride Crystals Group Ltd., Saint Petersburg, 194156, Russian Federation

Corresponding author: Natalia. Shmidt@mail.ioffe.ru

Article info

Received 04.12.14, accepted 20.12.14

doi: $10.17586 / 2226-1494-2015-15-1-46-53$

Article in Russian

Reference for citation: Shmidt N.M., Usikov A.S., Shabunina E.I., Chernyakov A.E., Kurin S.Yu., Makarov Yu. N., Helava H., Papchenko B.P. Study of mechanisms responsible for the efficiency degradation of the III-nitrides light emitting diodes. Scientific and Technical Journal of Information Technologies, Mechanics and Optics, 2015, vol. 15, no. 1, pp. 46-53 (in Russian) 
Abstract. The results for external quantum efficiency degradation of two types of light emitting diodes based on III-nitrides: blue and ultraviolet ones are presented. Existing mechanisms proposed for the degradation are considered briefly. Applying several techniques for studying the light emitting diodes at various stages of the aging test gives the possibility to reveal a new mechanism of defects formations with a help of multi-phonon recombination of carriers in an extended defects system and in local regions of random alloy fluctuations. These techniques include analysis of current voltage characteristics evolution at $\mathrm{V}<2 \mathrm{~V}$, the low frequency noise methods, and infrared microscopy. The multi-phonon recombination of carriers is accomplished by generation of native defects, in particular, In- or Ga-atoms and their migration. These processes lead to modification of the extended defects system properties and local composition of InGaN alloys in several regions that result in decreasing of the carriers participating in a radiative recombination and degradation of the external quantum efficiency. It was demonstrated that this mechanism of the defects formation can be responsible for the degradation of the blue and ultraviolet light emitting diodes. The mechanism can explain non monotonic dependence of the degradation process during the aging test, catastrophic failures of the light emitting diodes and low lifetime of the ultraviolet light emitting diodes.

Key words: light emitting diodes based on III-nitrides, system of extended defects, mechanisms of defects generation, degradation.

Acknowledgements. The work is performed by Saint Petersburg National Research University of Information Technologies, Mechanics and Optics and supported by the Ministry of Education and Science of the Russian Federation within the grant agreement \#14.575.21.0054 (unique identifier of research activities RFMEFI57514X0054) on the topic: «Study of new technical possibilities for the creation of clean hydrogen generator using a photoelectrochemical cell based on III nitride group semiconductor nanostructures».

\section{Введение}

Светодиоды на основе нитридов третьей группы, таких как InGaN/GaN и AlGAN/GaN - гетероструктуры, работающие в синем и ультрафиолетовом (УФ) спектральных диапазонах соответственно, находят обширное применение в различных областях человеческой деятельности. Наиболее впечатляющие успехи достигнуты в индустрии синих InGaN/GaN светодиодов, а ученые I. Akasaki, H. Amano и $\mathrm{S}$. Nakamura, заложившие научные основы этого направления, отмечены Нобелевской премией по физике за 2014 год. Однако очевидно, что далеко не все фундаментальные проблемы, препятствующие наиболее полной реализации потенциальных возможностей светодиодов на этих материалах, решены на сегодняшний день [1]. Многолетняя практика позволила на отдельных фирмах повысить срок службы синих InGaN/GaN светодиодов до 50000 часов, но катастрофические отказы по-прежнему повышают стоимость готового изделия (светодиодных ламп) и препятствуют полному переходу на энергосберегающее твердотельное освещение. Срок службы лучших УФ AlGaN/GaN светодиодов не превышает 2000 часов, что препятствует их применению в медицине для дезинфекции и стерилизации, для биотехнологий, в спектроскопии, в банковском деле для выявления фальшивых купюр, для полной замены ртутных ламп на экологически чистые твердотельные источники [2]. Особенностью деградации внешней квантовой эффективности (ВКЭ) синих светодиодов, установленной многими исследователями, является тот факт, что увеличение плотности тока больше $10 \mathrm{~A} / \mathrm{cm}^{2}$ при проведении экспериментов по старению, а также повышение температуры окружающей среды увеличивают скорость деградации оптической мощности [1]. Основным процессом, ответственным за катастрофическую деградацию светодиодов, некоторые авторы считали [3] электромиграцию ионов металла из контактов по протяженным дефектам в активную область. Однако такой вывод противоречит результатам исследований температурных зависимостей вольтамперных характеристик (BАХ) до и после деградации [4], что генерируемые при деградации дефекты, характеризуются теми же уровнями, что и до деградации. Эти авторы отметили нестабильное и частично обратимое поведение тока и оптической мощности перед катастрофической деградацией. Следует отметить, что эти особенности наблюдаются и на современных высокоэффективных синих и УФ светодиодах. Авторы ряда работ связывали деградацию оптической мощности InGaN/GaN светодиодов в первую очередь с изменением сопротивления $p$-области, индуцированным процессами интердиффузии и межфазных химических реакций [5], механическими напряжениями [6]. Важная роль во многих работах отводится трансформации водородосодержащих комплексов под действием длительного протекания инжекционного тока $[1,6]$. В [7] предложена модель дополнительной активации магния в результате разрыва связи магний-водород в $p$-области горячими носителями, инжектированными в квантовую яму и дрейфующими в $p$-область в поле компенсированного слоя, между активной и $p$-областями. Этот процесс, происходящий на первой стадии старения при временах 100-800 ч, сопровождается ростом заряженных акцепторных центров в $p$-области, а при больших временах старения из-за дефектообразования вакансий азота в результате разрыва связей галлий-азот в активной области горячими носителями начинается уменьшение значений ВКЭ. Эта модель до сих пор пользуется популярностью. Однако она не объясняет немонотонного, волнообразного течения процесса деградации ВКЭ светодиодов на разных временных стадиях старения. Снижение ВКЭ в УФ светодиодах объяснено активацией прорастающих дислокаций вакансиями азота, усилением проводимости этих дислокаций и потерей части носителей [2], или миграцией алюминия [8]. Общим мнением можно считать, что деградация оптической мощности светодиодов развивается в результате генерации точечных дефектов в активной области под действием инжекционного тока, усиливающей безызлучательную рекомбинацию $[1,9]$. Однако усиление генерации точечных дефектов 
должно было бы приводить на финальной стадии, также как в светодиодах на основе традиционных А3В5 материалов, к скольжению, переползанию и мультипликации дислокаций с образованием дислокационной сетки в активной области светодиодов. Эти процессы не наблюдаются в светодиодах на основе нитридов третьей группы [10]. Более того, наблюдается влияние токов утечки, шунтирующих $p$ - $n$ переход, на распределение электролюминесценции по площади светодиода [6] и появление локальных областей перегрева на финальной стадии деградации $[11,12]$, а также миграция галлия и индия вдоль дислокаций [11] с выделением металлической фазы на поверхности [12]. В связи с этим был сделан вывод о диффузии точечных дефектов и примесей вдоль прорастающих дислокаций в активной области светодиодной структуры [10]. В недавнем обзоре [1] отмечалось, что единого общепринятого механизма (или сочетания нескольких механизмов), способного описать наблюдаемые особенности развития деградации ВКЭ светодиодов при старении и катастрофический выход из строя светодиодов, на сегодняшний день не предложено. Более того, не выяснено, в каких областях преимущественно развивается дефектообразование под действием инжекционного тока, какова роль флуктуаций состава твердого раствора в этом процессе, есть ли общие механизмы, ответственные за деградацию ВКЭ в синих и УФ светодиодах, в чем причина такой сильной разницы в сроках службы этих двух типов светодиодов.

Целью работы является выяснение общих и отличающихся механизмов, приводящих при старении этих двух типов светодиодов к деградации ВКЭ, а также причин низкого срока службы УФ AlGaN/GaN светодиодов.

\section{Образцы и экспериментальные методики}

УФ светодиоды на основе $\mathrm{AlGaN} / \mathrm{GaN}$ структур были выращены на сапфировых подложках диаметром 50,8 мм методом хлоридно-гидридной газофазной эпитаксии в реакторе горизонтального типа. Особенности эпитаксиального роста и дизайн структур были описаны ранее [13]. После выращивания структур методами стандартной фотолитографии формировались омические контакты, травились мезадиоды заданной геометрии, проводилась резка структур на чипы и сборка светодиодов. Собранные светодиоды с размерами светоизлучающей площади 850 мкм $\times 850$ мкм имели максимум электролюминесценции на длине волны 350-360 нм и ВКЭ 1,5\% при токе 20 мА. В качестве второго типа светодиодов были использованы коммерческие InGaN/GaN светодиоды с размерами светоизлучающей площади 950 мкм×950 мкм. Они имели максимум электролюминесценции на длине волны 450-460 нм и ВКЭ 40-45\% при токе 20 мА. Эксперименты по старению синих светодиодов проводились при плотности тока $35 \mathrm{~A} / \mathrm{cm}^{2}$ при температуре $100{ }^{\circ} \mathrm{C}$. Условия исследования деградации УФ светодиодов были более мягкими $\left(30 \mathrm{~A} / \mathrm{cm}^{2}\right.$ и $45^{\circ} \mathrm{C}$ ) по сравнению с таковыми для синих светодиодов, но все же более жесткими по сравнению с режимами, обычно используемыми для такого типа светодиодов (3-4 A/cм² и комнатная температура).

Большая часть исследований проводилась после финальной стадии деградации, когда величина ВКЭ светодиодов уменьшалась на $30 \%$ относительно значений до старения. На этой стадии исследовалось изменение ВАХ зависимостей ВКЭ и дифференциального сопротивления светодиодов от плотности тока по сравнению с этими же характеристиками и зависимостями до старения. Кроме того, применялись методы исследования низкочастотного шума, которые, как известно, позволяют получить интегральную информацию не только о точечных дефектах, но и о флуктуациях зарядов и высоты барьеров, о степени однородности протекания тока, о присутствии областей локального перегрева [14]. Анализ низкочастотного шума проводился в диапазоне частот от 1 Гц до 10 кГц. Спектральная плотность шума измерялась при плотностях тока $10^{-4}-50 \mathrm{~A} / \mathrm{cm}^{2}$ для всех светодиодов. Следует отметить, что наиболее полные результаты получены на InGaN/GaN светодиодах.

\section{Результаты и обсуждение}

Вид ВАХ синих и УФ светодиодов (прямые и обратные ветви приведены в одном квадранте, значения тока $I$ и напряжения $U$ в вольтах приведены по абсолютной величине) при напряжении менее 2 В (рис. 1) свидетельствует, согласно [15], о шунтировании $p$ - $n$-перехода. Причем для того и другого типа светодиодов на финальной стадии наблюдается рост проводимости шунтов на порядки, относительно значений до старения.

При этом коммерческие светодиоды от разных производителей могут отличаться по величине проводимости шунтов более чем на порядок до старения. Ранее было показано, что проводимость шунтов или ток утечки, измеренный при напряжении менее 2 В, отражает интегрально свойства системы протяженных дефектов InGaN/GaN светодиодов [16]. При этом, чем меньше величина токов утечки, тем меньше разупорядоченность наноматериала и менее развита система протяженных дефектов. Причем не обнаружено явной зависимости между проводимостью шунтов и общей плотностью дислокаций. Этот вывод находит подтверждение в работах других авторов. Выяснено, что только часть протяженных дефектов являются проводящими. Это $V$-дефекты (ограненные поры, формирующиеся на дислокациях или скоплениях примесей, каплях металлической фазы), дефекты упаковки, прорастающие дислокации с ядром, содержащим металлические связи [17]. При этом, как показали исследования зависимостей спек- 
тральной плотности низкочастотного шума от плотности тока InGaN/GaN светодиодов, свойства шунтов изменяются нелинейно с ростом плотности тока [18]. В области плотностей тока больше 5 A/см² наблюдается рост уровня низкочастотного шума, и характер роста указывает на ухудшение свойств шунтов [18]. Если бы проводимость шунтов была омической, то наблюдаемый рост проводимости этих шунтов при напряжении менее 2 В после старения не должен был проявляться при больших плотностях тока, в силу малости их величины по сравнению с рабочими токами. Однако сравнение зависимостей ВКЭ от плотности тока светодиодов до и после старения выявляет рост проводимости шунтов при больших плотностях тока, что проявляется в сдвиге максимума этих зависимостей после старения (рис. 2) в сторону больших плотностей тока.
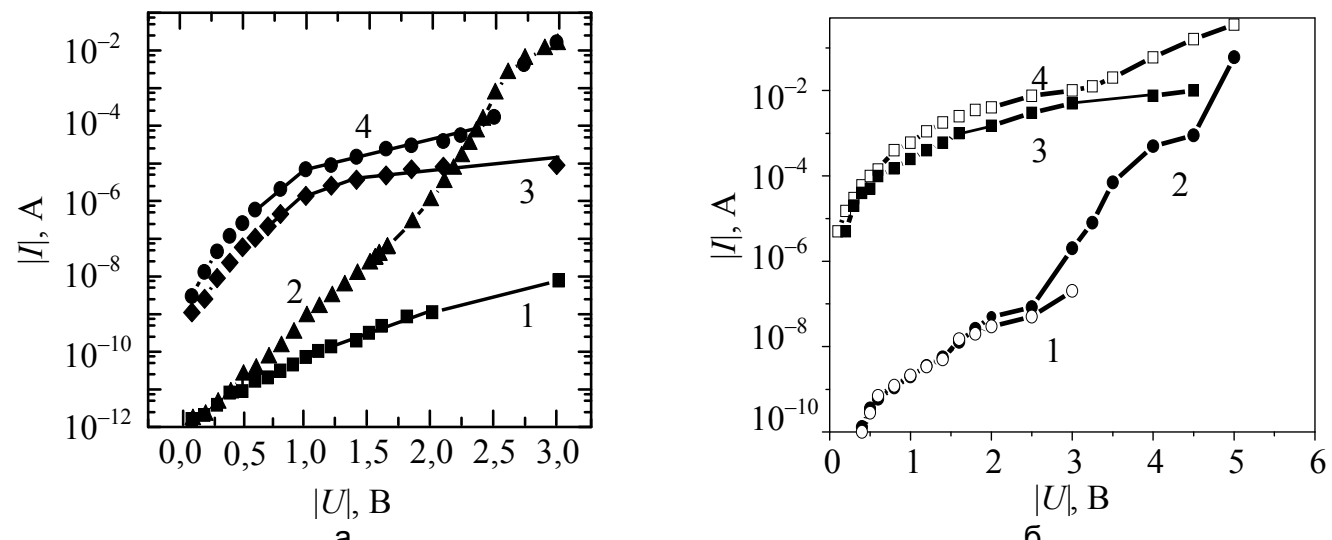

Рис. 1. Экспериментальные BAX светодиодов: InGaN/GaN (а) и AIGaN/GaN (б) до (кривые 1, 2) и после (кривые 3, 4) деградации. Обратные ветви BAX (кривые 1, 3), прямые ветви (кривые 2, 4)

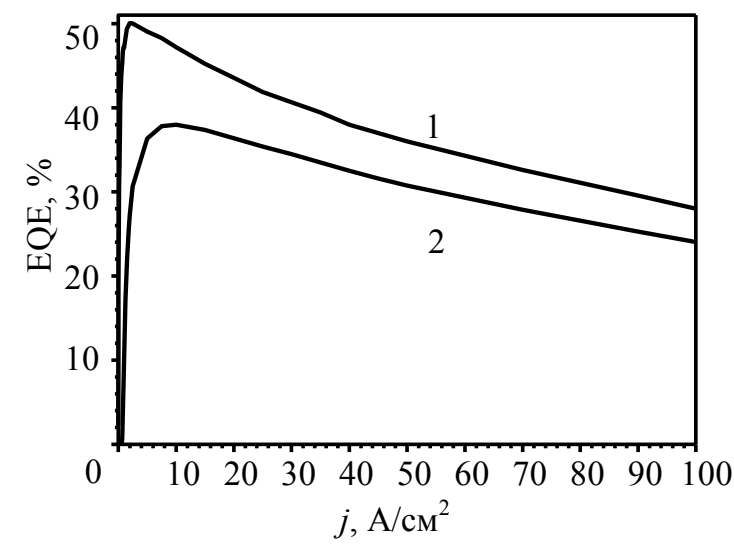

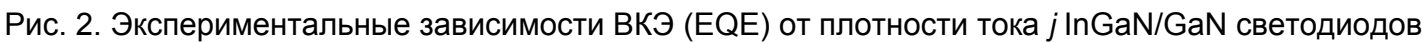
до (кривая 1) и после (кривая 2) финальной стадии старения

Наблюдаемый эффект свидетельствует об изменении проводимости шунтов под действием инжекционного тока. Этот эффект наиболее ярко проявляется на коммерческих InGaN/GaN светодиодах. Рост проводимости шунтов приводит к уходу носителей и к уменьшению максимума ВКЭ, что наблюдается для обоих типов исследуемых светодиодов. Аналогичные результаты для $\mathrm{AlGaN} / \mathrm{GaN}$ светодиодов приведены в [2]. Увеличение проводимости шунтов после финальной стадии старения усиливает неоднородное протекание тока, что выявляется по отклонению зависимостей плотности флуктуации напряжений $S_{V}$ от плотности тока $j$ (рис. 3) для тех и других светодиодов от зависимости $S_{V} \sim j^{-1}$, типичной для $p$ - $n$-структур с однородным распределением тока [8]. Причем неоднородность протекания тока как до, так и после старения наиболее ярко выражена для $\mathrm{AlGaN} / \mathrm{GaN}$ светодиодов (рис. 3, а).

Усиление неоднородности протекания тока приводит к перегреву локальных областей, которые выявляются методами инфракрасной микроскопии. Пример выявления таких областей в InGaN/GaN cветодиодах приведен на рис. 4. Оценка плотности тока, протекающего через шунты, дает величину, близкую к $1000 \mathrm{~A} / \mathrm{cm}^{-2}$. Как известно, высокая плотность тока является одним из условий возникновения многофононной рекомбинации носителей на глубоком возбужденном центре [19]. В качестве таких возбужденных центров в исследованных светодиодах могут выступать слабо связанные атомы индия и галлия, присутствующие в таких протяженных дефектах, как $V$-дефекты, дефекты упаковки, часть винтовых дислокаций с ядром, обогащенным атомами Ga или In. 


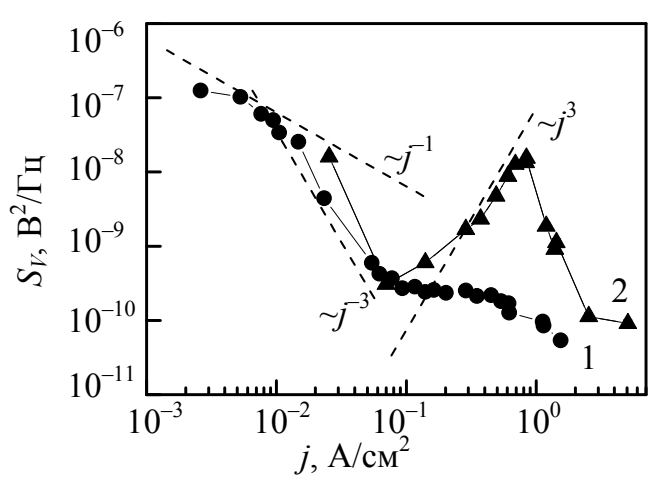

a

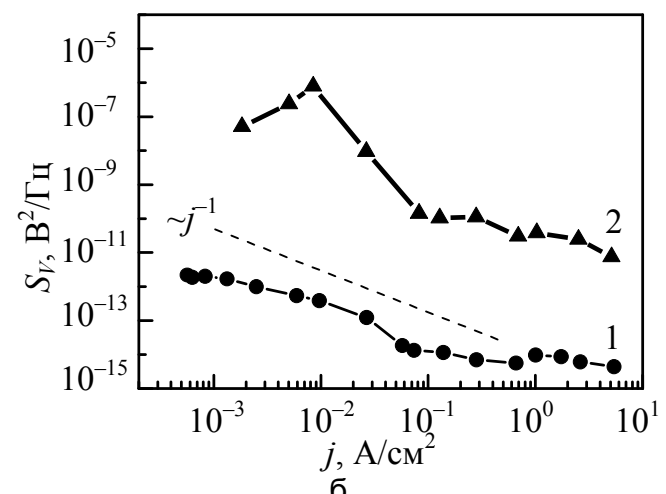

6

Рис. 3. Экспериментальные зависимости плотности фллукуации напряжений $S_{V}$ от плотности тока $j$ для светодиодов: AlGaN/GaN (a) и InGaN/GaN (б), до (кривые 1) и после (кривые 2) финальной стадии старения. Пунктирные линии представляют аппроксимацию экспериментальных данных степенными зависимостями $S_{V} \sim j^{-n}(n=1,3,-3)$, выявляющими различные механизмы протекания тока. Расчетная зависимость $S_{V} \sim j^{-1}-$ характерная для однородного распределения тока по площади светодиода

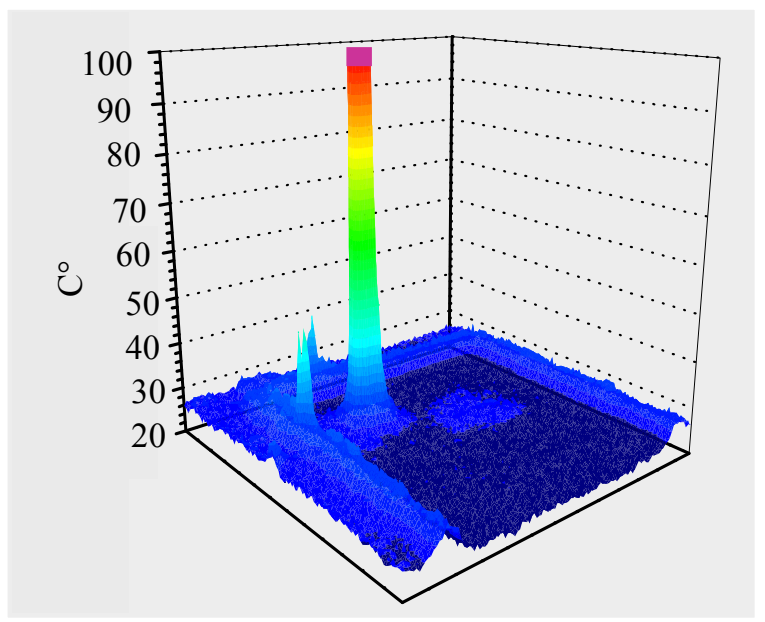

Рис. 4. Области локального перегрева в синих $\operatorname{lnGaN/GaN~светодиодах,~выявленные~методом~}$ инфракрасной микроскопии. Размер светоизлучающей площади 980×980 мкм

Захват дырок на такие центры, согласно [19], сопровождается локальным разогревом и смещением центра. Этот процесс вызывает образование дефектов по Френкелю и их миграцию. Подтверждением возможности развития этого процесса являются экспериментальные данные, полученные как в этой работе, так и другими исследователями на InGaN/GaN светодиодах, по выявлению миграции индия вдоль прорастающих дислокаций методами просвечивающей микроскопии [11] и по выделению индия и галлия в локальных областях на поверхности светодиодов [12] после финальной стадии старения.

Таким образом, процесс дефектообразования под действием инжекционного тока, изменяющий свойства шунтов, развивается локально в некоторых типах протяженных дефектов. Общность в изменении проводимости шунтов, локализованных в системе протяженных дефектов двух типов светодиодов после финальной стадии деградации, позволяет предполагать аналогичный механизм дефектообразования и в $\mathrm{AlGaN} / \mathrm{GaN}$ светодиодах. Кроме этого канала дефектообразования, в InGaN/GaN светодиодах был выявлен еще один канал. Присутствие этого канала было обнаружено впервые на светодиодах с катастрофическим выходом из строя (т.е. полным отсутствием излучения) при временах старения на порядки ниже типичных для финальной стадии [20]. Выяснено, что для таких светодиодов типичными являются искаженные прямые ветви ВАХ, примеры таких ветвей светодиодов из одной партии (кривые 2-5), но с близкой обратной ветвью (кривая 1) приведены на рис. 5. Причем искаженные ветви наблюдаются на небольшой части светодиодов до старения или появляются после коротких времен старения 100-500 ч. Согласно [15], вид искаженной ветви ВАХ свидетельствует о присутствии локальных областей с пониженной высотой потенциальных барьеров из-за обогащения твердого раствора атомами индия. Причем площадь этих областей на 4-5 порядков меньше всей излучающей площади светодиода. 


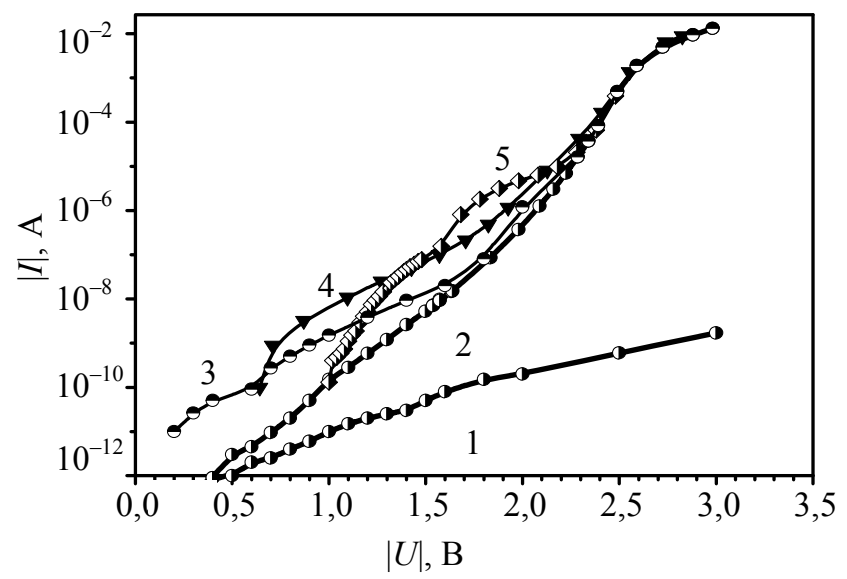

Рис. 5. Экспериментальные ветви ВАХ светодиодов: кривая 1 - обратная, одинаковая для всех светодиодов; прямые ветви - кривые 2-5. Для светодиодов с большим сроком службы прямые ветви близки к кривой 2. Примеры искаженных прямых ВАХ светодиодов с катастрофическим выходом из строя кривые 2-5

Исследование зависимостей спектральной плотности низкочастотного токового шума $\left(S_{I}\right)$ светодиодов от плотности тока $j$ показало, что в светодиодах с локальными пониженными барьерами (с искаженной прямой ветвью ВАХ) появляется участок сильной зависимости $S_{I} \sim j^{4}$ при малых токах $0,1-1$ мА, что указывает на возникновение областей локального перегрева [20]. Однако площадь областей с пониженными барьерами на порядки меньше всей площади светодиода, а это означает, что плотности тока в этих областях близки к $1000 \mathrm{~A} / \mathrm{cm}^{2}$. Следовательно, в этих областях возможно развитие многофононной рекомбинации носителей со всеми сопутствующими явлениями, такими как миграция атомов индия и галлия между локальными областями твердого раствора и перераспределение состава твердого раствора в них. Наиболее эффективно этот процесс может происходить в локальных областях с нерегулярным составом твердого раствора и приводить к небольшим изменениям значений ВКЭ как в сторону увеличения, так и в сторону уменьшения. Такое поведение хорошо согласуется с немонотонным изменением значений ВКЭ при старении светодиодов, а также с изменениями длинноволновой полосы с максимумом при 600 нм в спектрах электролюминесценции таких светодиодов [20].

На разных временных стадиях старения наблюдается чередование дефектообразования в системе протяженных дефектов и в локальных областях твердого раствора с пониженными барьерами (механизм один, области разные), и только в финальной стадии дефектообразования оба канала, как правило, участвуют одновременно, что видно по изменению прямой и обратной ветвей ВАХ при напряжениях менее 2 В. Типичным также является изменение только прямой ветви на ранних стадиях старения для светодиодов с катастрофическими отказами. В $\mathrm{AlGaN} / \mathrm{GaN}$ светодиодах искажение BAX является характерным для всех светодиодов до старения (рис. 1, кривая 2), что позволяет предполагать участие этого канала в дефектообразовании на всех стадиях старения. Однако более высокая проводимость шунтов, локализованных в системе протяженных дефектов, чем в InGaN/GaN светодиодах, осложняет разделение вклада этих двух каналов дефектообразования и изучение процессов, происходящих в локальных областях твердого раствора.

\section{Заключение}

Полученные результаты по изучению деградации внешней квантовой эффективности в двух типах светодиодов на основе нитридов третьей группы при их старении позволили предположить развитие многофононной рекомбинации носителей в части протяженных дефектов, обогащенных металлической фазой, и в локальных областях нерегулярного состава твердого раствора. Этот механизм приводит к локальному дефектообразованию собственных дефектов и позволяет объяснить единообразие дефектов, появляющихся на разных временных стадиях старения. Для этого механизма характерно волнообразное развитие дефектообразования во времени из-за возможности частичного залечивания ранее существовавших дефектов мигрирующими атомами Ga или In. Волнообразное развитие дефектообразования изменяет свойства шунтов и число инжектированных носителей, участвующих в излучательной рекомбинации, и, как следствие, величину внешней квантовой эффективности. Кроме общности этого механизма для синих InGaN/GaN и ультрафиолетовых $\mathrm{AlGaN} / \mathrm{GaN}$ светодиодов, выяснено, что проводимость шунтов, локализованных в системе протяженных дефектов, на несколько порядков выше на ультрафиолетовых $\mathrm{AlGaN} / \mathrm{GaN}$ светодиодах до старения, что и может являться одной из причин низких сроков службы этих светодиодов. Более сильные искажения прямых ветвей вольтамперной характеристики ультрафиолетовых $\mathrm{AlGaN} / \mathrm{GaN}$ светодиодов, чем синих $\mathrm{InGaN} / \mathrm{GaN}$, позволяют предполагать большую вероятность катастрофических отказов и требуют более детального изучения свойств AlGaN твердых растворов.

Научно-технический вестник информационных технологий, механики и оптики, 2015, том 15, № 1 


\section{References}

1. Meneghesso G., Meneghini M., Zanoni E. Recent results on the degradation of white LEDs for lighting. Journal of Physics D: Applied Physics, 2010, vol. 43, no. 35, art. 354007. doi: 10.1088/0022$3727 / 43 / 35 / 354007$

2. Pinos A., Marcinkevicius S., Shur M.S. High current-induced degradation of AlGaN ultraviolet light emitting diodes. Journal of Applied Physics, 2011, vol. 109, no. 10, art. 103108. doi: 10.1063/1.3590149

3. Meneghini M., Trevisanello L., Sanna C., Mura G., Vanzi M., Meneghesso G., Zanoni E. High temperature electro-optical degradation of InGaN/GaN HBLEDs. Microelectronics Reliability, 2007, vol. 47, no. 9-11, pp. 1625-1629. doi: 10.1016/j.microrel.2007.07.081

4. Bochkareva N.I., Rebane Yu.T., Gorbunov R.I., Klochkov A.V., Shreter Yu.G., Efremov A.A. Nonuniformity of carrier injection and the degradation of blue LEDs. Semiconductors, 2006, vol. 40, no. 1, pp. 118-123. doi: 10.1134/S1063782606010210

5. Hibbard D.L., Jung S.P., Wang C., Ullery D., Zhao Y.S., Lee H.P., So W., Liu H. Low resistance high reflectance contacts to p-GaN using oxidized Ni/Au and $\mathrm{Al}$ or Ag. Applied Physics Letters, 2003, vol. 83, no. 2. pp. 311-313. doi: $10.1063 / 1.1591233$

6. Fischer P., Christen J., Zacharias M., Schwegler V., Kirchner C., Kamp M. Spatially resolved imaging of the spectral emission characteristic of an InGaN/GaN-multi quantum well-light-emitting diode by scanning electroluminescence microscopy. Japanese Journal of Applied Physics, Part 1: Regular Papers and Short Notes and Review Papers, 2000, vol. 39, no. 4 B, pp. 2414-2416.

7. Kovalev A.N., Manyakhin F.I., Kudryashov V.E., Turkin A.N., Yunovich A.E. Changes in the luminescent and electrical properties of InGaN/AlGaN/GaN light-emitting diodes during extended operation. Semiconductors, 1999, vol. 33, no. 2, pp. 192-199.

8. Sawyer S., Rumyantsev S.L., Shur M.S., Pala N., Bilenko Yu., Zhang J.P., Hu X., Lunev A., Deng J., Gaska R. Current and optical noise of GaN/AlGaN light emitting diodes. Journal of Applied Physics, 2006, vol. 100, no. 3, art. 034504. doi: 10.1063/1.2204355

9. Cao X.A., Sandvik P.M., LeBoeuf S.F., Arthur S.D. Defect generation in InGaN/GaN light-emitting diodes under forward and reverse electrical stresses. Microelectronics Reliability, 2003, vol. 43, no. 12, pp. 19871991. doi: 10.1016/j.microrel.2003.06.001

10. Takeya M., Mizuno T., Sasaki T., Ikeda S., Fujimoto T., Ohfuji Y., Oikawa K., Yabuki Y., Uchida S., Ikeda M. Degradation in AlGaInN lasers. Physica Status Solidi C: Conferences, 2003, no. 7, pp. 2292-2295. doi: $10.1002 / \mathrm{pssc} .200303324$

11. Leung K.K., Fong W.K., Chan P.K.L., Surya C. Physical mechanisms for hot-electron degradations in GaN light-emitting diodes. Journal of Applied Physics, 2010, vol. 107, no. 7, art. 073103. doi: 10.1063/1.3357312

12. Kamanin A.V., Kolmakov A.G., Kop'ev P.S., Onushkin G.A., Sakharov A.V., Shmidt N.M., Sizov D.S., Sitnikova A.A., Zakgeim A.L., Zolotareva R.V., Usikov A.S. Degradation of blue LEDs related to structural disorder. Physica Status Solidi (C) Current Topics in Solid State Physics, 2006, vol. 3, pp. 2129-2132. doi: $10.1002 /$ pssc. 200565162

13. Kurin S., Antipov A., Barash I., Roenkov A., Usikov A., Helava H., Ratnikov V., Shmidt N., Sakharov A., Tarasov S., Menkovich E., Lamkin I., Papchenko B., Makarov Y. Characterization of HVPE-grown UV LED heterostructures. Physica Status Solidi (C) Current Topics in Solid State Physics, 2014, vol. 11, no. 3-4, pp. 813-816. doi: 10.1002/pssc.201300459

14. Chernyakov A.E., Levinshtein M.E., Talnishnikh N.A., Shabunina E.I., Shmidt N.M. Low-frequency noise in diagnostics of power blue InGaN/GaN LEDs. Journal of Crystal Growth, 2014, vol. 401, pp. 302-304.

15. Schubert E.F. Light-Emitting Diodes. Cambridge University Press, 2006, 327 p.

16. Averkiev N.S., Chernyakov A.E., Levinshtein M.E., Petrov P.V., Yakimov E.B., Shmidt N.M., Shabunina E.I. Two channels of non-radiative recombination in InGaN/GaN LEDs. Physica B: Condensed Matter, 2009, vol. 404, no. 23-24, pp. 4896-4898. doi: 10.1016/j.physb.2009.08.252

17. Cao X.A., Teetsov J.M., D'Evelyn M.P., Merfeld D.W., Yan C.H. Electrical characteristics of InGaN/GaN light-emitting diodes grown on GaN and sapphire substrates. Applied Physics Letters, 2004, vol. 85, no. 1, pp. 7-9. doi: 10.1063/1.1767280

18. Shabunina E., Averkiev N., Chernyakov A., Levinshtein M., Petrov P., Shmidt N. Extended defect system as a main source of non-radiative recombination in InGaN/GaN LEDs. Physica Status Solidi (C) Current Topics in Solid State Physics, 2013, vol. 10, pp. 335-337. doi: 10.1002/pssc.201200656

19. Yassievich I.N. Recombination-induced defect heating and related phenomena. Semiconductor Science and Technology, 1994, vol. 9, no. 8, pp. 1433-1453.

20. Chernyakov A.E., Levinshtein M.E., Petrov P.V., Shmidt N.M., Shabunina E.I., Zakgeim A.L. Failure mechanisms in blue InGaN/GaN LEDs for high power operation. Microelectronics Reliability, 2012, vol. 52, no. 9-10, pp. 2180-2183. doi: 10.1016/j.microrel.2012.06.051 
Шмидт Наталия Михайловна

Усиков Александр Сергеевич

Шабунина Евгения Игоревна

Черняков Антон Евгеньевич

Курин Сергей Юрьевич

Макаров Юрий Николаевич

Хелава Хейкки Илмари

Папченко Борис Петрович

Natalia M. Shmidt

Alexander S. Usikov

Evgeniya I. Shabunina

Anton E. Chernyakov

Sergei Yu. Kurin

Yuri N. Makarov

Heikki I. Helava

Boris P. Papchenko
- доктор физико-математических наук, старший научный сотрудник, главный научный сотрудник, ФТИ им. А.Ф. Иоффе, Санкт-Петербург, 194021, Российская Федерация, Natalia. Shmidt@mail.ioffe.ru

- кандидат физико-математических наук, технический директор, Нитридные кристаллы Inc-DP, Дир Парк, NY 11729, CША; соруководитель лаборатории, Университет ИТМО, Санкт-Петербург, 197101, Российская Федерация, alexander.usikov@nitride-crystals.com

- $\quad$ кандидат физико-математических наук, научный сотрудник, ФТИ им. А.Ф. Иоффе, Санкт-Петербург, 194021, Российская Федерация, Jenni-85@mail.ru

- $\quad$ научный сотрудник, ФТИ им. А.Ф. Иоффе, Санкт-Петербург, 194021, Российская Федерация, chernyakov.anton@yandex.ru

- ведущий специалист, ООО «Группа компаний «Нитридные кристаллы», Санкт-Петербург, 194156, Российская Федерация, sergey.kurin@nitride-crystals.com

- $\quad$ кандидат физико-математических наук, президент, Нитридные кристаллы Inc-DP, Дип Парк, NY 11729, США; генеральный директор, ООО «Группа компаний «Нитридные кристаллы», Санкт-Петербург, 194156, Российская Федерация, yuri.makarov@nitride-crystals.com

- $\quad \mathrm{PhD}$, вице-президент, Нитридные кристаллы Inc-DP, Дип Парк, 11729 , NY, СШA, heikki.helava@nitride-crystals.com

- $\quad$ начальник научно-технического отдела, Университет ИТМО, СанктПетербург, 197101, Российская Федерация, b.p.papchenko@gmail.com

- D.Sc., senior scientific researcher, Head researcher, Ioffe Institute, Saint Petersburg, 194021, Russian Federation, Natalia.Shmidt@mail.ioffe.ru $\mathrm{PhD}$, Chief technical officer, Nitride Crystals Inc-DP, Deer Park, NY 11729, USA; Co-director of Laboratory, ITMO University, Saint Petersburg, 197101, Russian Federation, alexander.usikov@nitridecrystals.com

- PhD, scientific researcher, Ioffe Institute, Saint Petersburg, 194021, Russian Federation, Jenni-85@mail.ru

- $\quad$ scientific researcher, Ioffe Institute, Saint Petersburg, 194021, Russian Federation, chernyakov.anton@yandex.ru

- $\quad$ Principal specialist, Nitride Crystals Group Ltd., Saint Petersburg, 194156, Russian Federation, sergey.kurin@nitride-crystals.com

- PhD, President, Nitride Crystals Inc-DP, Deer Park, NY 11729, USA; Director General, Nitride Crystals Group Ltd, Saint Petersburg, 194156, Russian Federation, yuri.makarov@nitride-crystals.com

- $\quad$ PhD, Vice-president, Nitride Crystals Inc-DP, Deer Park, NY 11729, USA, heikki.helava@nitride-crystals.com

- Head of Scientific and Technical Department, ITMO University, , Saint Petersburg, 197101, Russian Federation, b.p.papchenko@gmail.com 